\title{
AN INQUIRY-BASED FRESHMAN BIOCHEMISTRY LAB SET TO ENHANCE STUDENTS'AUTONOMY\#
}

\author{
Thanuci Silva e Eduardo Galembeck* \\ Departamento de Bioquímica e Biologia Tecidual, Universidade Estadual de Campinas, 13083-862 Campinas - SP, Brasil
}

Recebido em 17/10/2016; aceito em 02/03/2017; publicado na web em 29/03/2017

\begin{abstract}
Inquiry-based laboratory sessions are recognized for contributing to enhancing students' autonomy and they were used in the reorganization of the Biochemistry laboratory offered to freshman Biology students in this university. Students were previously asked to follow rigid laboratory protocols attempting to achieve the correct results of their experiments. The inquiry-based activities were designed to develop students autonomy to plan and to perform experiments, as well as communicating and discussing their results. The inquiry-based and autonomy approach followed is classified in the literature as Organizational, Procedural, and Cognitive. The levels of autonomy required from students were increased sequentially. The first activity was the least demanding, since the students received detailed instructions from the worksheet. The activities became gradually more complex, transferring to students most decisions on setting up and performing the experiments. Student skill improvement was obtained, as verified by excerpts from student reports, by data obtained from content analysis of the exams, and scientific report scores indicate that this approach may have stimulated the improvement of several skills related to different autonomy aspects. Most important, students showed increased ownership of the laboratory materials, reagents, laboratory environment and especially of learning, showing a sense of active participation in the experimental activities.
\end{abstract}

Keywords: inquiry-based; biochemistry laboratory; autonomy.

\section{INTRODUCTION}

Inquiry-based learning is an effective approach to enhance students' autonomy. ${ }^{1-8}$ Providing them fewer directions about the experimental design ${ }^{1,2}$ while posing the pertinent questions stimulates their thinking, their interest on the theoretical basis of their work and engages them in the development of the experimental protocols. ${ }^{3}$

On the other hand, students using strict laboratory protocols or "cookbook" laboratory styles always face difficulties to transfer the learned skills to new situations. Moreover, it is recognized that students are then led to choose easier ways to have their work done, contributing to low achievement. ${ }^{4,5}$

For several years, we based our activities on experimental protocols that were a predefined sequence of techniques intended to have students collecting data and checking if their findings were right or wrong. In agreement with several considerations made in the American Society for Biochemistry and Molecular Biology (ASBMB), ${ }^{6}$ and the "Vision and Change" report, ${ }^{7}$ we decided to reform the course curriculum to include inquiry-based lab activities $^{8}$. Our laboratory activities were also structured using autonomy categories classified as Organizational, Procedural, and Cognitive,, 5 varying the activities in levels of autonomy required from students.

The first activity was the simplest, fully instructed by worksheet containing all the information the students need to perform the experiments. From there on, students were progressively required to make more and more decisions on how to progress in their investigations, choosing among the techniques available in the laboratory to solve the research questions proposed to them and deciding on how to discuss and report their results.

*e-mail: eg@unicamp.br

\#This paper was presented in the SBQ - ACS symposium on Chemical Education, held in Goiânia, on May 2016. Publication costs were assisted by INCT Inomat, CNPq process 573644/2008-0

\section{METHODOLOGY}

We developed inquiry-based experiments for a Biochemistry of Proteins course, where students attend four hours of teaching sessions per week. The course was a traditional laboratory, focused on the development of abilities related to instrumentation and techniques performance without leaving much room for decisions or inputs from the students.

The course reformulation occurred in three moments. In the past, all the activities were fully instructed by the worksheets. After the first reformulation, the activities demanded a high level of autonomy from students, which made them struggle to make decisions by themselves. Then, in the second reformulation, we decided to develop activities of growing complexity. They evolved from simple tasks fully instructed in the worksheets, to multifaceted ones requiring self-guidance. The aim was to have students engaged in the solution of problems while developing their autonomy.

The successive activities grew more intricate as the course progressed, both considering the topics addressed and the level of autonomy required from students.

Three steps composed all the activities. Planning sessions were used by students to develop their working plans. At the end of each session, each group of students presented to the instructors their plans for the upcoming sessions, written in their laboratory notebooks.

Next, the experimental sessions were dedicated to data collection. Students worked together in groups of 8 students sharing and organizing the laboratory tasks while paying attention to the plan previously drawn. Lastly, the groups engaged in analyzing the acquired results, interpreting and discussing them, considering the literature.

At the end of each activity, students were required to hand a report structured as a scientific report that was used to measure how well they exerted their autonomy. This aspect is also designed to develop their ability to communicate and to structure their experimental findings.

Before starting each subsequent (and more complex) activity, they learned their research report scores, including comments about the faculty expectations on their level of achievement. 
Students also took partial examinations during the semester that allowed faculty to assess each students' performance.

\section{RESULTS AND DISCUSSION}

In this work, we discuss the data obtained from the second course reformulation. The autonomy support used to develop the activities was based on three categories of autonomy support. It consists of varying essential levels of engagement, in such aspects as Organizational, Procedural, and Cognitive ${ }^{5,9}$ (Table 1). We then reorganized the activities, increasing these levels of engagement gradually over the time. Based on students' degrees of freedom to decide on experiment directions, the curriculum was then reorganized in three lab activities.

The first activity, Buffering Systems, has a worksheet equipped with all the information students need to perform the experiments. The goals, the research questions, and the hypotheses were given to them. It requires low levels of the autonomy aspects mentioned above (Table 1). It means, students do not have many opportunities to decide on the experiments directions, since it is driven by the activity worksheet. In this activity, they needed to simulate a titration in an Excel worksheet and to perform it in the laboratory, identifying possible causes for the differences found, between the simulation and the titration performed.

We analyzed the discussion portion of the scientific report, and we observed that many students failed to interpret their data and attempted to repeat the information provided in the worksheet to justify the differences between the simulation and the titration performed. Below is an example obtained from a group report (Figure 1).

\section{"Errors from team execution}

There may have been errors arising from the working inability of the executors, such as for controlling the burette valve and adding the exact titrant aliquot, burette reading errors, and variations of the reading parameters between the executors, since we took turns among the group members to perform triplicates."

As observed in Figure 1, the discussion mentioned above is not what their actual data represents. Students also used other information from activity worksheet to explain the graph shown in Figure 1, such as errors related to equipment imprecision and unexpected solutions behavior.

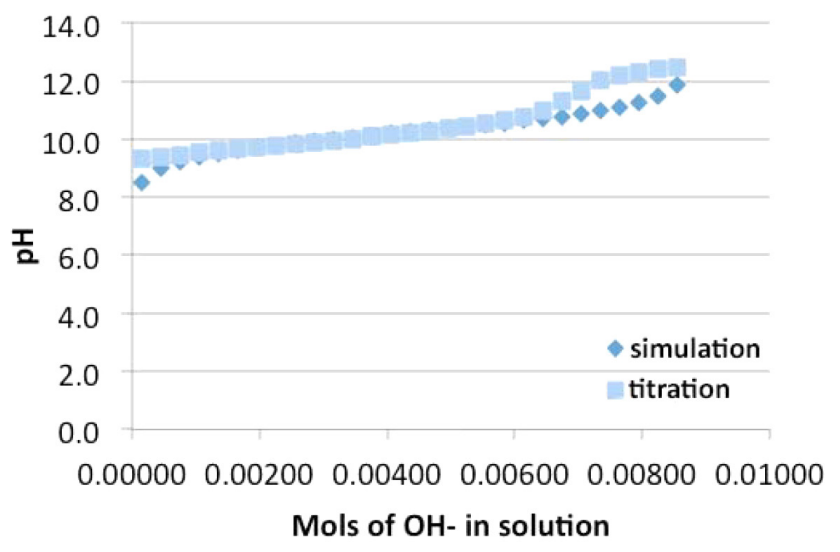

Figure 1. Example of data collected from a student group report of Activity 1 (Buffering Systems)

We included a similar graph in the Buffering Systems exam, and the answers followed the same pattern observed in the scientific reports. A content analysis of the respective exam question showed that none of the students had a performance rated as Great, while $44 \%$ of students had a performance rated as Regular and $56 \%$ of students had a performance rated as Bad.

According to studies in the field of students' autonomy, when instructors provide many directions about the experiment, students tend to cut corners to have their work done. ${ }^{4,5}$ In our case, students decided to rewrite the information given in the activity worksheet instead of looking at their actual data, discuss with peers and find their explanations to the phenomena observed.

Furthermore, deciding on the need for preparing new reagents and the need for collecting further data are related to the Organizational aspect of autonomy, ${ }^{5}$ which encourages students ownership of the laboratory environment. Although these autonomy features introduces to students the meaning of control, the long-lasting effects of autonomy that we seek to promote may not have been set.

Based on data from scientific reports, we observed that students performance significantly increased $(\mathrm{p}<0.05)$ from Activity 1 to Activity 2 (Figure 2). ${ }^{8}$ Although Activity 1 was the less complex activity proposed to students regarding autonomy and topics addressed, we observed that this was also the source of the lowest student grades in this course. In this sense, we also understand from the teaching experience that sometimes students struggle with the first activities proposed, since they do not know what the instructors

Table 1. Autonomy Support proposed by Stefanou ${ }^{5}$ and $\mathrm{Wu},{ }^{9}$ used to reform the laboratory activities (adapted from ref. 8 )

\begin{tabular}{|c|c|c|c|}
\hline Autonomy Support & Autonomy Category & $\begin{array}{l}\text { Lab Activities before } \\
\text { course reform }\end{array}$ & $\begin{array}{l}\text { Lab Activities after } \\
\text { course reform }\end{array}$ \\
\hline Choosing group members & Organizational & High & High \\
\hline Deciding on the need for preparing new reagents & Organizational & Low & High \\
\hline Deciding on the need for collecting further data & Organizational & Medium & High \\
\hline Creating their own activity protocol & Procedural & Low & High \\
\hline Choosing the reagents and techniques to be used & Procedural & Low & High \\
\hline Choosing the way to present the strategy (flowcharts, tables, list of items) & Procedural & Low & High \\
\hline Handling reagents, equipment and laboratory materials & Procedural & High & High \\
\hline Discussing different strategies to carry out the experiments with peers & Cognitive & Low & High \\
\hline Finding multiple ways (to solve the problem proposed & Cognitive & Low & High \\
\hline Collecting experimental data in groups & Cognitive & Low & High \\
\hline Reviewing literature to support data found & Cognitive & Low & High \\
\hline Drafting a report & Cognitive & Low & High \\
\hline
\end{tabular}




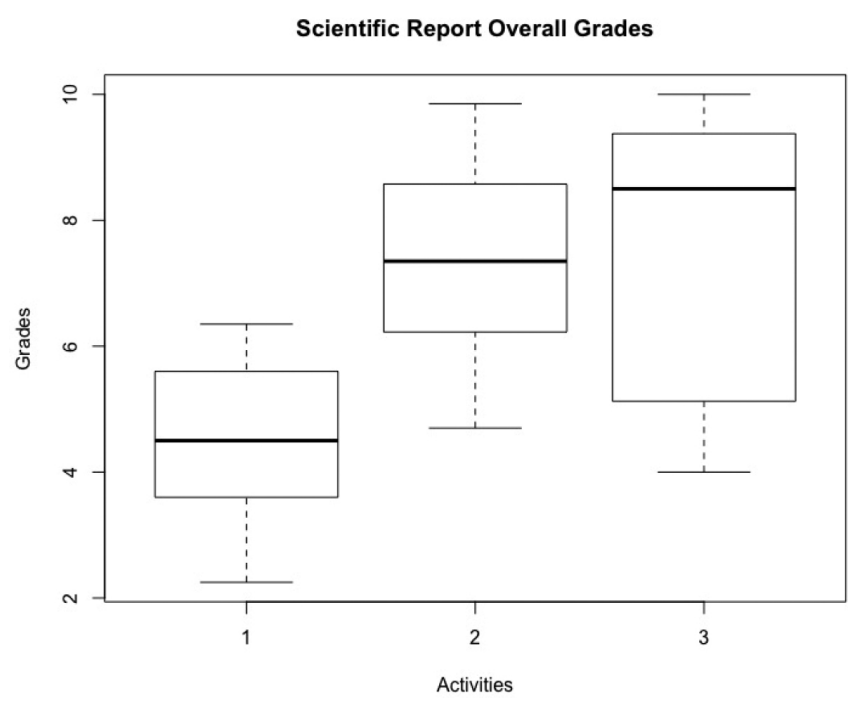

Figure 2. Overall grades of scientific reports returned at the end of each activity proposed. ANOVA analysis of variance was performed $\left(p=2.8 \times 10^{-5}\right)$ and then Fisher's LSD test, where we observed significant differences between the grades of Activity 1 and Activity $2\left(p=1.1 \times 10^{-4}\right)$ Activity 1 and Activity $3(p=$ $\left.2.6 \times 10^{-5}\right)$. Between Activity 2 and Activity 3 we did not observe a significant difference $(p=0.65)$

are expecting in their reports, even though they provide the rubrics for assessing them.

The second activity is called Amino Acids and Proteins. This activity aimed to gather as many information as possible from the samples provided. This activity represents a more complex situation where students need to develop by themselves a strategy using the techniques and reagents available. Amino Acids and Proteins activity brings mostly low and medium levels of the autonomy aspects (Table 1).

Students were told that the samples provided might have either only proteins or amino acids, or mixed proteins, or none of them (water). In the activity worksheet, students had information about the experimental techniques available, as well as the goals of the experiment and its hypothesis.

Analyzing the content of the reports, we may observe their improvement on discussing the data found, considering that in this activity they collected data from three unknown techniques for them: electrophoresis, paper chromatography, and Bradford protein assay.

"Using the Bradford protein assay, we identified samples containing proteins: A and C. However, it was still necessary to obtain more information about these and other samples: $B$ and $D$.

Then, we performed paper chromatography (...). In this sense, we could conclude that sample A contained only proteins since the paper chromatography did not detect the presence of amino acids. This technique also informed us that samples $B, C$, and contained amino acids. Therefore, we can say that Sample $C$ contained a mixture of proteins and amino acids, whereas $B$ and $D$ contained only amino acids.

With the results obtained from the method of Bradford and the paper chromatography, we were able to answer the questions initially raised: Sample A contains only proteins; Sample B and $D$ contains only amino acids; Sample C contains proteins and amino acids.

However, it was still possible to obtain more information about samples containing amino acids: what were these amino acids?
Performing a titration brought us some amino acid possibilities. We chose Sample D to proceed with Titration because it showed only one Retention Factor value on its paper chromatography, so possibly it had a single amino acid (unlike Sample B, whose paper chromatography indicated the presence of two amino acids).

The titration graph obtained informed us buffer $p K a$ which, when compared with other $p K a$ values tabulated for amino acids, helped us to reach a more refined result: Sample D quite possibly contains the amino acids Serine or Alanine, whose titration curves are very similar to the titration curve of sample D."

In the exam question covering the topic Amino Acids and Proteins, we asked students how to determine the concentration of an unknown protein. The content analysis revealed that $29 \%$ of the students had their answer rated as Great, which means they were able to describe how the Bradford protein assay can give the data necessary to determine the concentration of an unknown protein. In another hand, $56 \%$ of the students analyzed were rated as Regular, which means they were able to choose the Bradford protein assay to determine the proteins' concentration, although they were not able to describe how to perform it. Moreover, $15 \%$ of the students were rated as $\mathrm{Bad}$, which means that they mentioned techniques that do not fit in the sense of providing information about proteins concentration. Compared to Bufering Systems exam rates, these data may also represent an evidence of students' autonomy improvement.

The abilities to create an activity protocol and choosing the reagents and techniques to be used, features widely explored in Acitivty 2, are related to the Procedural aspect of autonomy, ${ }^{5}$ which encourages students ownership of materials and presentation forms.

The third and last activity proposed is called Enzymes. This activity aimed to develop an experimental plan to investigate the differences between two phosphatases and to define the enzymatic parameters of each one. This is the most complex activity introduced because only the experimental goals and information about the reagents and techniques available were given to students, and enzymes were the most complex topic taught to them. It presents mostly medium and high levels of autonomy engagement (Table 1). They needed to come up not only with experiment's strategy but also, find multiple solutions to solve the problem posed and define the experiment hypothesis.

Below, there is an excerpt from a scientific report, an evidence that students may have improved their ability to plan and perform the experiments proposed, compared to the other activities.

"In different organisms, enzymes have different structural
forms and respond differentially to their substrates. Iden-
tifying such differences, we evaluated the specificity of the
phosphatases with the substrate. First, an aliquot with diffe-
rent concentrations of pea and bean enzymes was extracted
and added to the same amounts of substrate (pNPP). Then,
the optimal concentration for measuring the enzymatic action
was chosen. The quantity of product formed was then mea-
sured, and this value was divided by the reaction time. With
these values, graphical representations of Michaelis-Menten
and Lineweaver-Burk were constructed, which allowed the
calculation of the 'Km' and the 'maximum speed reaction.'
With these values, we calculated the enzymatic specificity."

In the exam covering Enzymes, we asked students how they could experimentally discover the $\mathrm{Km}$ and the Vmax of an enzymatic reaction. The results showed that $31 \%$ of students had a performance 
rated as Great, which means they were able to describe in detail how to obtain a Lineweaver-Burk graph to determine $\mathrm{Km}$ and Vmax. We rated $28 \%$ of the students as Regular, which means that they either used Michaelis-Menten graph to determine the kinetics parameters or they made mistakes with the Lineweaver-Burk graph axis. Moreover, $41 \%$ of students were rated as Bad, which means students failed in to describe the process to discover the required kinetics parameters.

We observed an increase in the number of answers rated as Regular and Bad from Activity 2 to Activity 3, also data from scientific reports (Figure 2), shows that there is not significant improvement in their scores from Activity 2 to Activity 3, although there is significant difference from the scores of Activity 1 to Activity 3.

Thus, this approach is capable of stimulating the improvement of several skills related to different autonomy aspects. Planning, interpreting, identifying inconsistent components of the experiments, to discuss different strategies to carry out the experiments and drafting a scientific report are opportunities to exercise the Cognitive support of autonomy, ${ }^{5}$ which encourages students' ownership of learning. In this sense, the cognitive aspect of autonomy is recognized as promoting not only greater involvement of students but enduring the motivation and engagement.

\section{CONCLUSIONS}

The inquiry-based approach and the autonomy support adapted to our laboratory activities improved long-lasting skills associated to autonomy and laboratory experimentation, enhancing their capability to drive the activities forward and to make decisions by themselves. It is also capable of stimulating students' confidence to transfer the theoretical knowledge to experimental practices and to take the responsibility for their experimental design.
With the introduction of inquiry-based experiments, our students seem to grow more confident about the decisions taken the laboratory. This approach leads students to autonomously reflect about the rationale underlying the problems and the research questions proposed, providing a sense of active participation.

\section{ACKNOWLEDGEMENTS}

São Paulo Research Foundation (FAPESP) - Process: 2014/244320 - for financially supporting this work

\section{REFERENCES}

1. Cunningham, S. C.; McNear, B.; Pearlman, R. S.; Kern, S. E.; CBE-Life Sciences Education 2006, 5, 281.

2. Kuldell, N. H.; CBE-Life Sciences Education 2006, 5, 247.

3. Domin, D. S.; J. Chem. Educ. 1999, 76, 109.

4. Chan, V.; Teaching in Higher Education 2010, 6, 37.

5. Stefanou, C. R.; Perencevich, K. C.; DiCintio, M.; Turner, J. C.; Educational Psychology 2004, 39, 97.

6. Voet, J. G.; Bell, E.; Boyer, R.; Boyle, J.; Leary, M. O.; Zimmerman, J. K.; Biochem. Mol. Biol. Educ. 2003, 31, 161.

7. Anderson, C. W.; Bauerle, C.; DePass, A.; Donovan, S.; Drew, S.; Ebert-May, D.; Gross, L.; Hoskins, S. G.; Labov, J.; Lopatto, D.; Lynn, D.; McClatchey, W.; Varma-Nelson, P.; O'Connor, C.; Pelaez, P.; Poston, M.; Singer, S.; Tanner, K.; Wessner, D.; White, H.; Withers, M.; Wood, W.; Wubah, D. In Vision and Change in Undergraduate Biology Education. A Call to Action; Brewer, C. A., Smith, D., eds.; American Association for the Advancement of Science (AAAS): Washington, DC, 2009.

8. Silva, T.; Galembeck, E.; J. Chem. Educ. 2016, 94, 52.

9. Wu, J.; CBE-Life Sciences Education 2013, 12, 460. 